\title{
Optimal Sampling Designs for Monitoring Plant Frequency
}

\author{
John S. Heywood ${ }^{1}$ and Michael D. DeBacker ${ }^{2}$ \\ Authors are ${ }^{1}$ Professor, Biology Department, Missouri State University, Springfield, MO 65897; and ${ }^{2}$ Ecologist, Heartland Network Inventory and \\ Monitoring Program, National Park Service, Republic, MO 65802.
}

\begin{abstract}
Plant frequency is a pragmatic surrogate for plant density in protocols designed for the long-term monitoring of diverse communities. Frequency estimates are based on presence/absence data from plots of fixed size, and plots are usually spatially aggregated into sites (often transects) to reduce field effort. Using a combination of statistical models and computer simulations, we identify sampling designs that maximize statistical power for detecting changes in underlying plant density based on the analysis of plant frequency. The optimal plot size for collecting frequency data decreases both with increasing spatial variation in local density (spatial structure) and with increasing numbers of plots per site. Over realistic ranges for these parameters, plots of optimal size yield mean frequencies that vary from $20 \%$ to $80 \%$. However, with the exception of highly overdispersed populations, power is relatively insensitive to plot size; consequently, a plot size that yields a mean frequency of $50 \%$ usually provides nearly maximal power. For population monitoring, in which comparisons are made between successive samples from the same population, repeated measures from fixed sites improve statistical power substantially if there is spatial structure among sites, provided that the spatial pattern is at least partially consistent over time. However, there is still a power loss to the extent that the pattern of spatial structure among sites changes over time (a site-by-time interaction). This power loss can be mitigated by increasing the spacing between plots within sites, which has the effect of increasing the within-site structure and reducing the between-site structure. With more than 1 plot per site, there is no statistical advantage to obtaining repeated measures from fixed plots; relocating plots within sites in successive samples may therefore be advisable to minimize disturbance to the community.
\end{abstract}

\begin{abstract}
Resumen
La frecuencia de vegetación es una alternativa pragmática a la densidad de vegetación en el monitoreo a largo plazo en comunidades diversas. Las mediciones de frecuencia se basan en presencia o ausencia de vegetación en parcelas de un tamaño determinado, donde éstas parcelas estan a su vez confinadas dentro de un espacio de dimensiones estándar (generalmente un transecto) para asi facilitar el trabajo de campo. Utilizando una combinación de modelos estadisticos y simulaciones computarizadas, hemos identificado los diseños de muestreo que maximizan la capacidad de los métodos estadísticos para detectar cambios en densidad de la vegetación en base a análisis de frecuencia de vegetación. Las dimensiones óptimas de la parcela donde se van a recolectar datos de frecuencia disminuyen tanto con la variacion espacial en densidad local (estructura espacial) como con el numero de parcelas por sitio de muestreo. Algunas cifras reales de estos parámetros indican que las dimensiones normales de una parcela arrojan frecuencias con valores entre un $20 \%$ y $80 \%$. Sin embargo, con la exepción de publaciones sumamente dispersas, la capacidad estadística es relativamente insensible a las dimensiones de la parcela y, por consecuencia, una pacela que produce una frecuencia promedio del $50 \%$ generalmente provee una capacidad muy cercana a la cifra máxima. Para monitoreo de poblaciones en las cuales se realizan comparaciones entre muestras sucesivas para la misma poblacion, la medición repetida de sitios de muestreo fijos incrementa la capacidad estadistica considerablemente, pero solamente si existe una estructura espacial entre sitios de muestreo y suponiendo que el patrón espacial es suficientemente consistente con respecto al tiempo. Sin embargo, existe todavía la posibilidad de que la capacidad estadística se reduzca si el patrón de estructura espacial cambia con respecto al tiempo (una interacción sitio-tiempo). Esta pérdida en la capacidad estadística se puede mitigar al incrementar el espacio entre parcelas dentro del sitio de muestreo, el cual tiene el efecto de incrementar la estructura dentro del sitio de muestreo y de reducir la estructura dentro de éste. El tener mas de una parcela por sitio no afecta la ventaja estadística esperada por tener mediciones repetidas de parcelas fijas; sin embargo cambiando las parcelas de lugar dentro del sitio de muestreo en muestreos sucesivos es recomendable ya que asi se minimizan disturbios a la comunidad.
\end{abstract}

Key Words: plant density, plot size, spatial structure, statistical power

\section{INTRODUCTION}

Research was funded in part by the National Park Service through the Upper and Middle Mississippi Valley Cooperative Ecosystem Studies Unit, task agreement J6370031003.

Correspondence: John Heywood, Biology Department, Missouri State University, 901 South National Avenue, Springfield, MO 65897. Email: johnheywood@missouristate.edu

Manuscript received 9 September 2006; manuscript accepted 4 May 2007.
The long-term monitoring of a diverse plant community requires the repeated acquisition of data on abundance and distribution from a large number of species. The effort required to obtain density data on such a scale is usually not practical, thereby necessitating the use of less informative indicators of abundance. One such indicator that has been widely used is 
plant frequency, defined as the fraction of equal-area sample plots in which a species is present. Frequency data are often recommended for plant population monitoring because they are easy to obtain and are relatively stable in the face of seasonal and interannual environmental fluctuations (Hyder et al. 1966; Mueller-Dombois and Ellenberg 1974; Greig-Smith 1983; Kershaw and Looney 1985; Mosley et al. 1986; Smith et al. 1986, 1987; Causton 1988; Bonham 1989; Lesica and Hanna 2002). In the present study we examine the statistical efficiency of frequency data and identify optimal sampling designs for the collection of frequency data.

Sites where frequency data are to be collected must be placed at random within the study area in order to ensure a representative sample. Because of the substantial effort involved in locating and moving between random sites, most field studies designed to record frequency data have used a hierarchical sampling design, with multiple plots spatially aggregated into each site, often along transects (e.g., Hyder et al. 1965; Mosley et al. 1986; Smith et al. 1986; Hartnett and Fay 1998; Peet et al. 1998; DeBacker et al. 2004). Thus, within the constraints of a balanced, nested design, there are 3 design features that can be adjusted when collecting frequency data: the number of randomly located sites $(n)$, the number of frequency plots per site $(m)$, and the size of each plot (as measured by the mean number of plants per plot, $d$ ).

An optimal sampling design for collecting frequency data will maximize statistical efficiency (statistical power or, equivalently, estimation confidence) under the constraint of a fixed total effort devoted to data collection. For any particular species, the global optimum would be obtained by maximizing statistical power simultaneously with respect to $n$, $m$, and $d$. However, there are several pragmatic arguments for performing 2 separate optimizations, 1 with respect to $n$ and $m$ and the other with respect to $d$ under the constraint of fixed $n$ and $m$. First, the amount of field effort required to collect frequency data is strongly affected by both the number of sites $(n)$ and the number of plots per site $(m)$ but is less strongly affected by plot size $(d)$. As a consequence, the constraint of a fixed total effort will have a strong effect on the optimization of $n$ and $m$ but a weaker effect on the optimization of $d$. Second, simultaneous optimization of all 3 parameters may lead to species-specific values for $n$ and $m$, a design that is not practical to implement. A more practical approach is to estimate single, consensus values for $n$ and $m$ to be used for all species and then employ several different plot sizes that span the range of values that are optimal (given $n$ and $m$ ) for the set of species being studied.

The problem of optimizing $n$ and $m$ has been addressed by Hyder et al. (1963). For a fixed total number of plots, frequency sampling is most efficient when plots are randomly distributed within the study area, that is, when $m=1$. However, when the total field effort is fixed, frequency sampling is most efficient when plots are aggregated into sites. When plots are spatially aggregated, the efficiency of frequency sampling is reduced if the mean local density of plants varies among sites (spatial structure). Hyder et al. (1963) derive values for $m$ and $n$ that maximize the sampling efficiency for plant frequency, as a function of the spatial variance in frequency among sites, the effort required to locate a site within the study area, and the additional effort required to find and record data from each plot within a site.

Several studies have addressed the problem of choosing an appropriate plot size for recording frequency data. Curtis and McIntosh (1950), Hyder et al. (1963), and Critchley and Poulton (1998) recommend plot sizes that yield particular intermediate frequencies or ranges of frequencies, but their recommendations are based on informal arguments rather than a formal analysis of statistical efficiency. Several authors have proposed that an improved frequency value can be obtained by combining frequency data obtained from plots of several different sizes. Morrison et al. (1995) used computer simulations to assess 2 such measures proposed by Outhred (1984) that combine frequency data from nonoverlapping plots of progressively increasing size. They demonstrated that these measures are sensitive to density changes over a broader range of densities and are less sensitive to nonrandom patterns of dispersion than are frequency values based on a fixed plot size. Smith et al. (1987) claimed that the sum of frequencies across a set of nested plots provides more statistical power than do frequency data from any one plot size. However, because the data obtained from nested plots are not independent, the chisquare test that these authors employed to analyze the summed data suffers from pseudoreplication and is therefore overly liberal. Even if their claim is correct, it may simply reflect the larger sample size (larger number of plots) represented by the summed data. Data from a set of plots of optimal size would surely provide greater power than an equal number of plots of variable size. However, combining data from plots of several sizes does have the advantage that the same field protocol can be applied to all species regardless of their densities.

The effect of plot size on the statistical efficiency of frequency data has not been formally analyzed. Whysong and Brady (1987) used computer simulations to examine statistical power, but they emphasized the effect of plot number rather than plot size and presented results for only 2 plot sizes (yielding frequencies of $20 \%$ and $50 \%$ ). Furthermore, they simulated frequencies directly rather than the underlying densities, and thus their approach cannot be used to assess the effect of plot size on the power to detect a given change in underlying density.

Variation in local density across the range of a study area (spatial structure) may affect the optimal plot size for recording frequency data, but to our knowledge this issue has not been addressed in the literature. If local density varies among sample plots, then the optimal plot size will vary among plots. In such cases it is desirable to choose a single plot size that is an optimal compromise among the locally optimal plot sizes. Previous authors who have proposed an optimal mean frequency per plot (Curtis and McIntosh 1950; Hyder et al. 1963; Critchley and Poulton 1998) have implicitly assumed that the globally optimal mean frequency is simply the arithmetic mean of the locally optimal mean frequencies, thereby rendering spatial structure irrelevant. This assumption is suspect and requires investigation.

Here we present an analysis of the effects of plot size and spatial structure on the statistical power provided by frequency data. An essential point that has not been raised in the literature is the fact that it is change in density rather than frequency that is of fundamental interest, and consequently the optimal 
frequency-based test is the one that maximizes power for detecting a specified change in density, not a specified change in frequency. Thus, although hypothesis testing will necessarily involve frequency comparisons, sampling designs and data analyses should be chosen so as to maximize the efficiency of inferences about underlying densities. Because the relationship between density and frequency is nonlinear, designs that are optimal for detecting changes in density are not optimal for detecting changes in frequency. However, for a given change in density, the resulting change in frequency is less likely to be detected if the test is optimized for detecting frequency changes than if it is optimized for detecting density changes. This is an important point that has been overlooked by many previous investigations of sampling designs for frequency estimation.

Thus, the purpose of the present study is to examine the statistical efficiency of frequency data, with respect to inferences on plant density, as a function of plot size, spatial structure in plant density, and the degree of spatial aggregation of plots. For given amounts of spatial structure and patterns of spatial aggregation of plots, we provide optimal plot sizes for drawing inferences on plant density from frequency data. We also review the relationship between statistical efficiency and the physical proximity of aggregated plots in order to provide a comprehensive set of recommendations for collecting frequency data.

\section{METHODS}

The model system for the power analysis includes a pair of study areas within which frequency plots are spatially aggregated into sites. Each plot yields a frequency value of 0 or 1 , and these data are used to draw inferences about the difference in mean density between the 2 areas. This design corresponds to a fully nested analysis of variance (ANOVA), with each plot providing 1 value for the response variable. Although this binary (Bernoulli) response variable is decidedly nonnormal, the computer simulations described below indicate that the ANOVA test for differences between study areas is extremely robust if the number of sites per study area $(n)$ and the number of plots per site $(m)$ are both at least 5 . For example, with $n=5$ and $m=5$, the actual type I error rate in $10^{6}$ Monte Carlo simulations was 0.0996 for a nominal type I error rate of 0.10 . If the number of plots per site is constant across all sites within both areas, so that nesting is fully balanced at the lower level, then the $F$ test for the area effect is identical to a 2-sample $t$ test in which the sample mean frequencies per site are used as the raw data. Balanced nesting is the usual practice for the collection of frequency data, so it is assumed for the present study. Consequently, all power analyses are based on the 2-sample $t$ test.

In order to find the plot size that maximizes statistical power for each of a wide range of spatial patterns in density, power must be determined for many different sets of parameter values. Although exact results can be obtained by computer simulation, the number of simulations called for would require prohibitive amounts of computer time. To avoid this problem, the plot size that maximizes statistical power was estimated by obtaining an approximate expression for the expected value of the test statistic for the $t$ test, $\mathrm{E}(T)$, and then finding the plot size that maximizes $\mathrm{E}(T)$. The reliability of this approach was assessed by comparison with direct computer simulations of statistical power. The approximation for $\mathrm{E}(T)$ was obtained by replacing each constituent estimator with its expected value, yielding

$$
\mathrm{E}(T) \approx \frac{\mu_{A}-\mu_{B}}{\sqrt{V\left(\bar{f}_{A}\right)+V\left(\bar{f}_{B}\right)}},
$$

where $\mu$ is the true mean frequency within a study area, $\bar{f}$ is the estimated mean frequency within a study area, and $V(\bar{f})$ is the true variance of the frequency estimator. To obtain an expression for $V(\bar{f})$, we note that the actual frequency ( 0 or 1) for the $j$ th plot (from 1 to $m$ ) within the $i$ th site (from 1 to $n$ ) can be written as the sum of independent terms, $f_{i j}=\mu+$ $s_{i}+p_{i j}$, where $\mu_{i}=\mu+s_{i}$ is the mean frequency in site $i$ and $f_{i j}$ $=\mu_{i}+p_{i j}$ is sampled from a Bernoulli distribution with mean $\mu_{i}$. Because site and plot frequencies are written as deviations from the mean, $E_{s}\left(s_{i}\right)=0$ and $E_{p}\left(p_{i j}\right)=0$, where $E_{s}(\cdot)$ is the expectation across sites and $E_{p}(\cdot)$ is shorthand for $E_{p}\left(\cdot||_{s}\right)$, the expectation across plots within a site. The quantity $E_{s}\left(s_{i}^{2}\right)=\sigma_{s}^{2}$ is the spatial variance in frequency among sites, and $E_{p}\left(p_{i j}^{2}\right)=\mu_{i}\left(1-\mu_{i}\right)$ is the Bernoulli variance. Using this notation, the standard variance decomposition for the nested design can be written as

$$
V(\bar{f})=\frac{\sigma_{s}^{2}}{n}+\frac{V(p)}{n m},
$$

where $V(p)$ is the variance in the value of the frequency deviation $p_{i j}$ among all possible plots of the designated size. Noting that

$$
\begin{aligned}
V(p) & =E_{s} E_{p}\left(p_{i j}^{2}\right) \\
& =E_{s}\left(\mu_{i}-\mu_{i}^{2}\right) \\
& =\mu-E_{s}\left(\mu+s_{i}\right)^{2} \\
& =\mu(1-\mu)-\sigma_{s}^{2},
\end{aligned}
$$

equation [2] can be written as

$$
V(\bar{f})=\frac{\mu(1-\mu)+(m-1) \sigma_{s}^{2}}{n m} .
$$

Inserting equation [4] into equation [1] provides an approximate expression for $\mathrm{E}(T)$ as a function of the mean $(\mu)$ and spatial variance $\left(\sigma_{s}^{2}\right)$ of plant frequency within each of the 2 study areas, the number of sampling sites $(n)$ per study area, and the number of frequency plots $(m)$ per site.

To express $\mathrm{E}(T)$ in terms of plant densities rather than frequencies, probability distributions must be specified for the variation in mean density across sites within a study area and for the variation in mean density among plots within a site. Parameters of the frequency distribution can then be expressed in terms of parameters of the density distribution. To make these expressions analytically tractable, both density distribu- 
tions were assumed to be uniform. More complicated distributions, such as a truncated normal or a beta distribution, might more closely approximate the actual spatial distribution of plant densities in any particular population. However, because of the monotonic relationship between density and frequency, spatial variance in density will be the major determinant of spatial variance in frequency, with the specific shape of the density distribution having only secondary effects. Although the conversion from density parameters to frequency parameters is tractable with uniform density distributions, the computations are complex and require numerical approximations. Descriptions of the algorithms that were used to perform these computations are posted at http://biology.missouristate. edu/faculty_pages/Heywood/Heywood.htm.

The magnitude of spatial structure in plant density at each scale is measured by the relative range of the uniform distribution of plant densities $\left(r_{p}\right.$ for structure among plots within sites, $r_{s}$ for structure between sites). Thus, if site $i$ has a mean density per plot of $d_{i}$, then within site $i$ the local densities vary uniformly from $d_{i}\left(1-r_{p}\right)$ to $d_{i}\left(1+r_{p}\right)$. Possible values for the relative density range within a site vary from a minimum of $r_{p}=0$ (no density structure within the site) to a maximum of $r_{p}=1$ (local densities vary uniformly from 0 to $2 d_{i}$ plants per plot). Similarly, if an entire study area has a mean density per plot of $d$, then the mean density per site varies uniformly from $d\left(1-r_{s}\right)$ to $d\left(1+r_{s}\right)$. Finally, on the scale of an individual plot, plants are assumed to be distributed at random in space so that the actual number of plants in a particular plot is sampled from a Poisson distribution.

With this model, the maximum possible value for the frequency variance among sites is $\sigma_{s}^{2}=0.0661$, which is obtained when $r_{s}=1, r_{p}=0$, and the mean frequency across all plots is $\mu=0.70$. This is not an unusually large amount of spatial structure. For example, 18 fixed sites within 2370 ha of the Tallgrass Prairie National Preserve (Kansas) have yielded estimates for $\sigma_{s}^{2}$ that range from 0.019 to 0.059 for 8 focal species (DeBacker and Heywood, unpublished data).

Statistical power was estimated by Monte Carlo simulation. For specified values of $r_{p}, r_{s}$, the percent difference in mean density between study areas, and the type I error rate for statistical testing, simulated data sets were generated and analyzed as follows. First, for each site within a study area, the mean density per plot, $d_{i}$, was sampled from a uniform distribution with mean $d$ and range $2 r_{s} d$, where $d$ was the mean density per plot for the entire study area. Second, for each plot within site $i$, the local density $d_{i j}$ was sampled from a uniform distribution with mean $d_{i}$ and range $2 r_{p} d_{i}$. Third, the actual number of plants in plot $j$ of site $i$ was sampled from a Poisson distribution with mean $d_{i j}$. Next, densities were converted to frequencies, and the sample mean frequency for each site was calculated. Finally, the simulated data set was analyzed using a 2-sample $t$ test, and the null hypothesis of equal mean frequencies in the 2 study areas was either rejected or not rejected. This entire process was repeated 100000 times. The power of the $t$ test is estimated as the fraction of these 100000 random data sets that led to rejection of the null hypothesis. All power simulations were conducted with a type I error rate of $10 \%$; this is an arbitrary choice and does not affect the sampling design features that maximize power.
The estimates of $\mathrm{E}(T)$ and the power simulations described above both assume that plants are randomly distributed within the vicinity of a plot. Plant populations sometimes have negative spatial structure on a local scale; that is, they are sometimes overdispersed relative to a Poisson distribution. To investigate the consequences of local overdispersion, power simulations were performed for a limited number of sets of parameter values. The simulations were as described above, except that each plot was assumed to be located within a local area where the plants were arranged uniformly into a square lattice with a spacing of $\sqrt{a / d_{i j}}$. Circular plots of area $a$ were located at random within this lattice to record frequency data. It was not possible to get a closed expression for $\mathrm{E}(T)$ with this sampling model.

All simulation programs were written in Fortran and were compiled in the MS Windows XP environment using MS Fortran Powerstation.

\section{RESULTS}

The plot size that maximizes statistical power is indistinguishable from the plot size that maximizes the approximate expected value of the $T$ statistic (Fig. 1). Thus, maximizing $\mathrm{E}(T)$ is an efficient

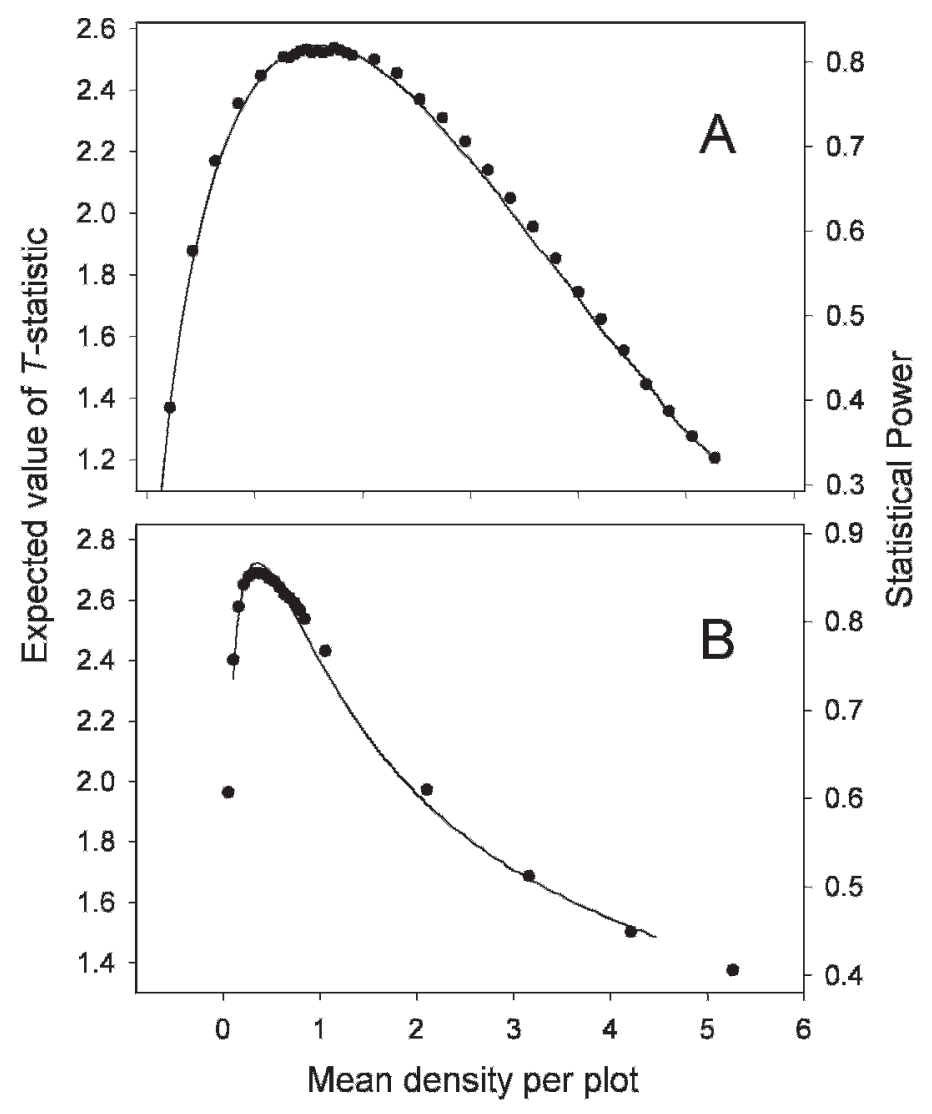

Figure 1. Estimated statistical power for $\alpha=0.1$ (symbols) and expected value of the $T$ statistic (curve) as a function of plot size for a 2sample $t$ test comparing the mean frequencies of 2 populations between which there is a $10 \%$ difference in mean density. $\mathbf{A}, r_{s}=r_{p}=0$ (no spatial structure), $n=100$, and $m=20$. B, $r_{s}=r_{p}=1$ (maximum spatial structure), $n=1000, m=20$. 

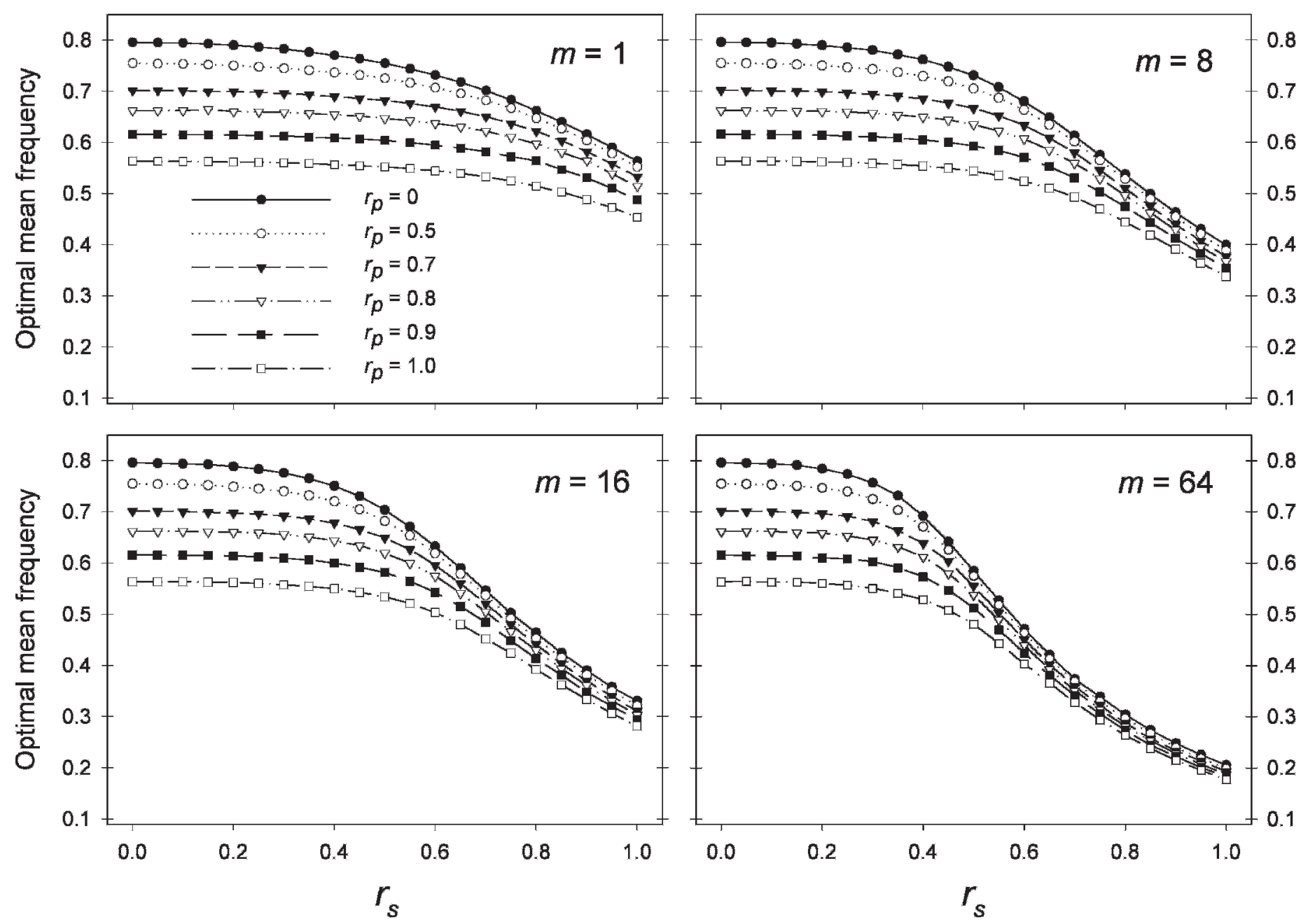

Figure 2. The mean frequency measured by plots of optimal size, as a function of the magnitude of spatial structure in density among plots within sites $\left(r_{p}\right)$, the magnitude of spatial structure in density among sites $\left(r_{s}\right)$, and the number of plots per site $(m)$.

way to identify the plot size that maximizes statistical power. This plot size will henceforth be referred to as the optimal plot size.

Figure 2 displays the optimal plot size (represented as the mean frequency per plot) as a function of the number of plots per site $(m)$, the range of density variation among sites $\left(r_{s}\right)$, and the range of density variation among plots within sites $\left(r_{p}\right)$. These optima were obtained by maximizing $\mathrm{E}(T)$ under the assumption that plants are randomly distributed in space within the vicinity of each plot. The optimal plot size decreases with increasing values of both $r_{s}$ and $r_{p}$ (Fig. 2). In the absence of spatial structure among sites $\left(r_{s}=0\right)$, the optimal plot size is not affected by the number of plots per site, with plots of optimal size yielding mean frequencies that range from a high of 0.798 when $r_{p}=0$ to a low of 0.563 when $r_{p}=1$. However, if there is spatial structure between sites $\left(r_{s}>0\right)$, then as the number of plots per site increases, the optimal plot size decreases, and the effect of structure within sites lessens. In the most extreme case that was considered $\left(r_{s}=1, m=64\right)$, the optimal plot size yields a mean frequency of only about $20 \%$, and this value is only weakly affected by the amount of spatial structure within sites (Fig. 2).
When plants are uniformly distributed within the vicinity of individual plots (i.e., plants are highly overdispersed on a local scale), power simulations indicate that the optimal frequency is always shifted toward a more intermediate value (closer to $50 \%$; Fig. 3). If, in addition, spatial structure is absent or weak, then power drops off much more rapidly as plot size deviates from the optimum than is the case when plants are randomly distributed on a local scale (Fig. 3A). However, this effect of overdispersion is virtually eliminated by a moderate amount of spatial structure (Figs. 3B-3D).

Figure 4 demonstrates the effects of spatial structure on statistical power when frequency data are always collected using the optimal plot size. Spatial structure within sites $\left(r_{p}>\right.$ $0)$ has very little effect on statistical power regardless of the number of plots per site (Figs. 4A and 4B). If frequency plots are not spatially aggregated into sites $(m=1)$, then spatial structure among sites $\left(r_{s}>0\right)$ likewise has very little effect on statistical power (Figs. 4C and 4D). However, when plots are spatially aggregated within sites, spatial structure among sites reduces power substantially, and this effect increases with increasing numbers of plots per site (Figs. 4C and 4D). 

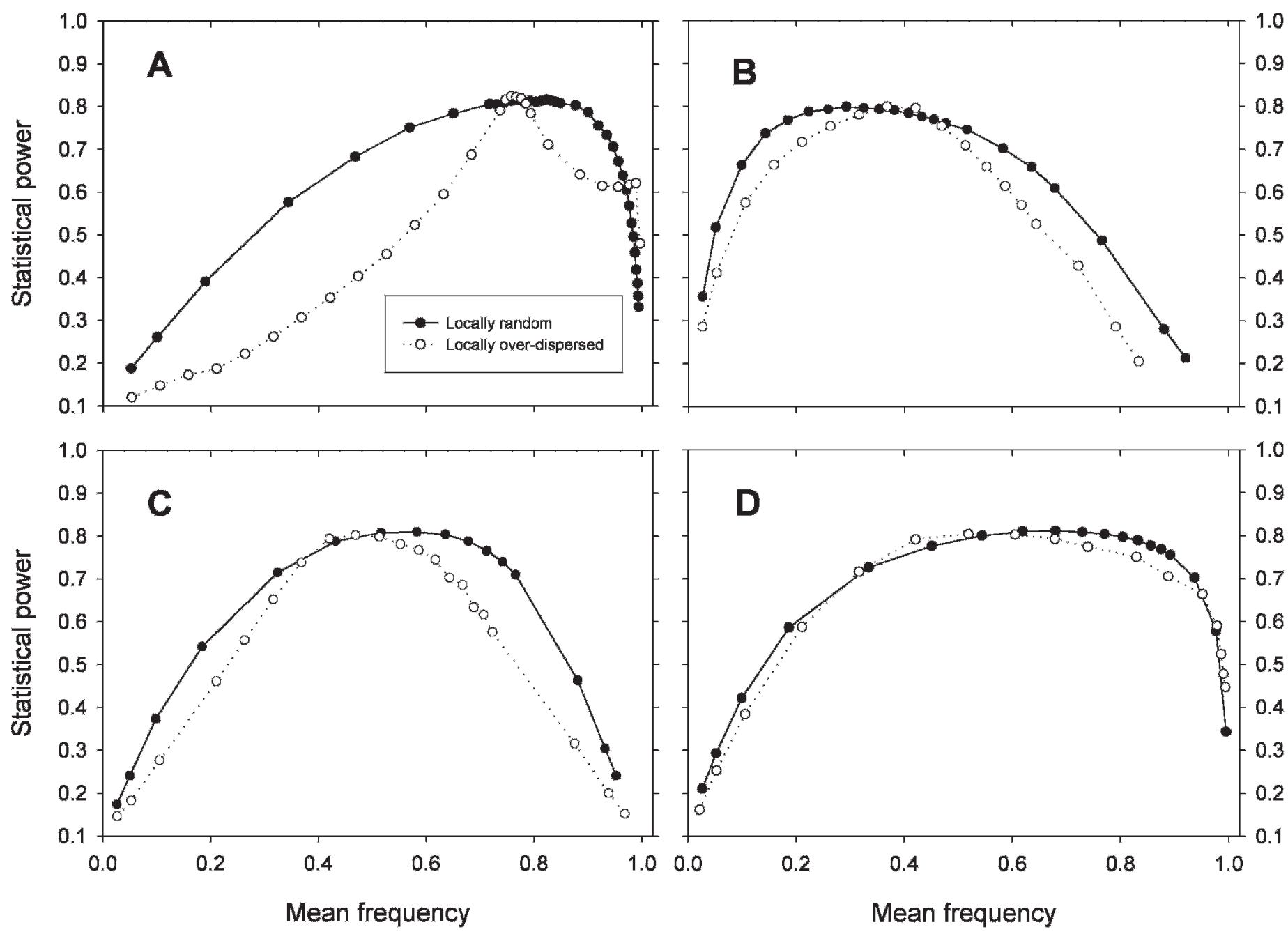

Figure 3. Estimated statistical power (for $\alpha=0.1$ ) as a function of plot size (represented by the mean frequency it measures) for a 2-sample $t$ test comparing the mean frequencies of 2 populations between which there is a $10 \%$ difference in mean density. In the vicinity of individual plots, plants were either distributed randomly in space (solid symbols) or highly overdispersed (open symbols). The number of plots per site was fixed at $m=20$. A, $r_{s}=r_{p}=0$ (no spatial structure). B, $r_{s}=1, r_{p}=0$ (structure between sites only). C, $r_{s}=0, r_{p}=1$ (structure within sites only). D, $r_{s}=r_{p}=0.5$ (modest structure at both scales).

\section{DISCUSSION}

\section{Plot Size}

Plot size affects both the numerator and the denominator of the $T$ statistic, and as a consequence the relation between plot size and statistical power is complex and not intuitively obvious. Plot size affects the numerator of the $T$ statistic because it affects the sensitivity of frequency to changes in density. The plot size that maximizes the sensitivity of frequency to changes in density and hence that maximizes the numerator of the $T$ statistic depends on the amount of spatial structure both within and between sites. With no spatial structure and a Poisson distribution of individuals within plots, the numerator is maximized when there is an average of 1 individual per plot, which corresponds to a mean frequency of 0.632 . This is the result that led Curtis and McIntosh (1950) to propose that the optimal plot size for collecting frequency data will yield a mean frequency of 0.63 . When spatial structure is present, the plot size that maximizes the sensitivity of frequency to changes in density will yield a mean frequency that is less than 0.632 , with the mean frequency decreasing as the magnitude of spatial structure increases.

Plot size also affects the denominator of the $T$ statistic, and as a consequence the plot size that maximizes statistical power can deviate considerably from the plot size that maximizes the sensitivity of frequency to changes in density. Plot size affects the denominator of $T$ because it affects the precision with which frequencies are estimated. The variance of a frequency estimate is proportional to $\mu(1-\mu)+(m-1) \sigma_{s}^{2}$, where $\mu$ is the actual mean frequency per plot and $\sigma_{s}^{2}$ is the spatial variance in mean frequency among sites (equation [4]). If there is no spatial structure among sites $\left(\sigma_{s}^{2}=0\right)$ or if plots are not spatially aggregated into sites $(m=1)$, then the frequency estimate is least precise (has the highest variance) when it is based on a plot size that yields a mean frequency of $\mu=0.5$, and precision increases symmetrically as plot size is either increased or decreased away from this value. When plots are aggregated within sites $(m>1)$, spatial structure among sites reduces the precision of the frequency estimate because data collected from multiple plots within the same site are correlated (pseudo- 

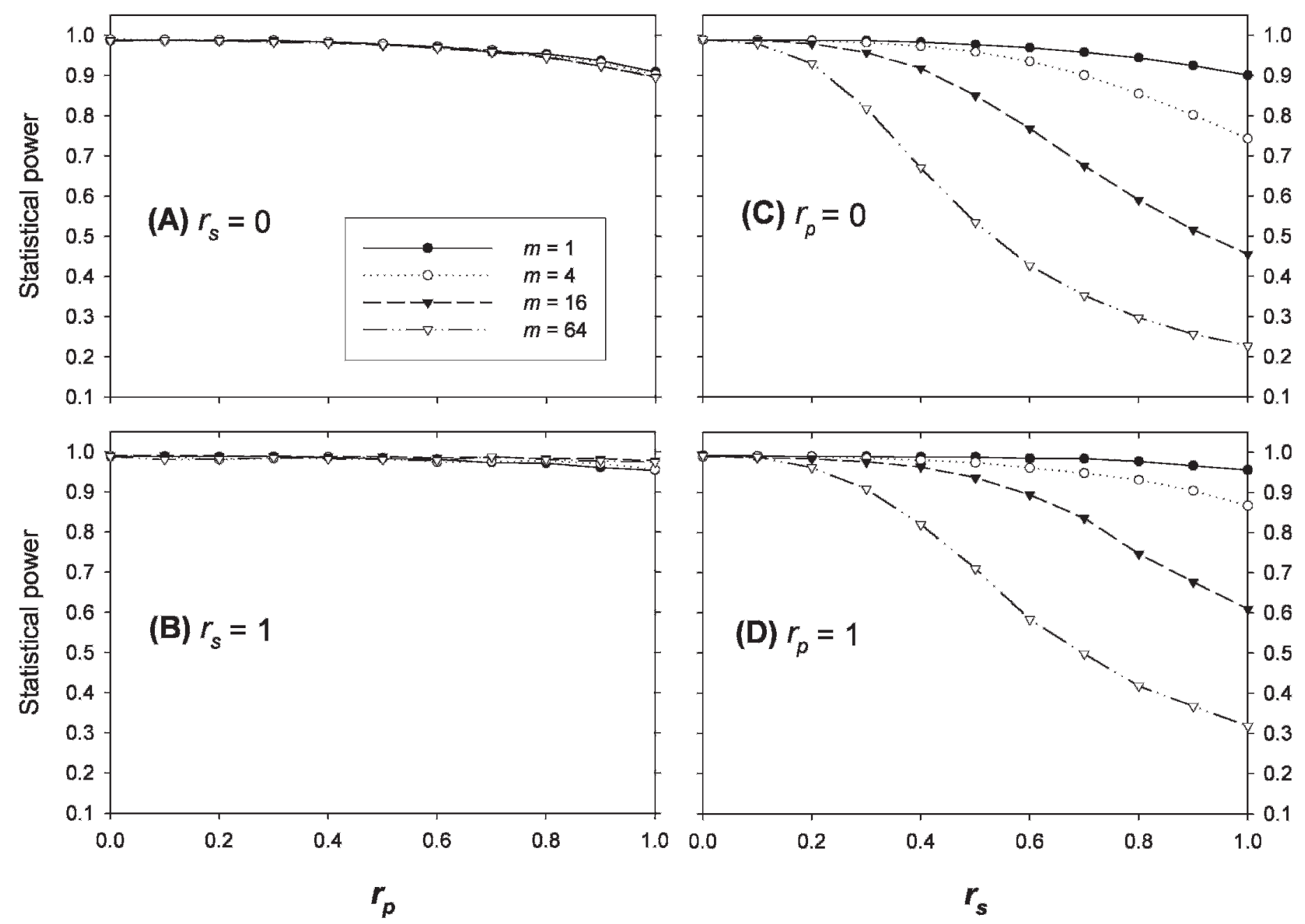

Figure 4. The effects of spatial structure $\left(r_{p}\right.$ and $\left.r_{s}\right)$ and the number of plot per site $(m)$ on estimated statistical power (for $\left.\alpha=0.1\right)$ when plots of optimal size are always used. For each power curve, the number of sites per population $(n)$ was adjusted so that power equals 0.99 when the abscissa equals 0 . A. Effect of structure within sites $\left(r_{p}\right)$ when there is no structure between sites $\left(r_{s}=0\right)$. B, Effect of structure within sites $\left(r_{p}\right)$ when there is maximum structure between sites $\left(r_{s}=1\right)$. C. Effect of structure between sites $\left(r_{s}\right)$ when there is no structure within sites $\left(r_{p}=0\right)$. D, Effect of structure between sites $\left(r_{s}\right)$ when there is maximum structure within sites $\left(r_{p}=1\right)$.

replication). The spatial variance in frequency, $\sigma_{s}^{2}$, and hence its contribution to the variance of the frequency estimate, will be maximal for a plot size that yields an intermediate mean frequency and will decrease toward 0 as plot size is either increased from this value (and $\mu$ approaches 1 ) or decreased from this value (and $\mu$ approaches 0 ). Thus, in general, the denominator of $T$ will be largest for plots that yield an intermediate mean frequency and will decrease asymmetrically toward 0 as the plot size is decreased or increased away from this intermediate value.

Combining these 2 effects of plot size, the $T$ statistic (and hence statistical power) is maximized by a plot size that yields the mean frequencies displayed in Figure 2. In the absence of spatial structure, plots of optimal size yield a mean frequency of 0.798 , considerably higher than the value of 0.62 proposed by Curtis and McIntosh (1950). The optimal plot size is reduced by spatial structure, and this effect is enhanced when plots are aggregated within sites. In the most extreme case that was considered $\left(r_{s}=1, m=64\right)$, the optimal plot size yields a mean frequency of less than 0.2 , a surprisingly low number.
Thus, the optimal plot size depends greatly on the amount of spatial structure in density, information that generally is not available when a monitoring protocol is being designed. Fortunately, however, statistical power is affected only weakly by deviations from the optimal plot size, with 1 exception. The exception occurs when plants are overdispersed in the vicinity of plots and spatial structure is weak or absent (Fig. 3A). This combination of features requires that the population be uniformly overdispersed at the same spatial scale over the entire study area. Intraspecific competition may generate overdispersion for dominant species within a community of very low diversity (e.g., Fonteyn and Mahall 1981), but such a pattern is unlikely for a population that is embedded within a community of high diversity with multiple codominant species. Furthermore, even if competition does generate local overdispersion, local density is unlikely to be uniform across the spatial extent of a management unit. Therefore, for most species, statistical power is nearly optimized across a broad range of plot sizes. In fact, over the entire range of possible values for $r_{p}$ and $r_{s}$, a plot size that returns a mean frequency of 
0.5 will provide nearly optimal power for most species (Fig. 3). Thus, in most communities, a sampling protocol that includes frequency plots of several different sizes spanning 1 to 2 orders of magnitude should include at least 1 plot size that yields statistical power close to the maximum that is possible for any particular species.

\section{Plot Arrangement}

Spatial structure has a strong effect on statistical power when plots are spatially aggregated into sites. Even if plots of optimal size are always used, power diminishes rapidly with increasing structure among sites (Figs. 4C and 4D). In contrast, spatial structure within sites has little effect on power (Figs. 4A and 4B). Thus, statistical power is greatly improved when all the spatial variation in density resides between plots within sites and none resides between sites. Spatial variation in plant density is an intrinsic feature of the population being monitored and cannot be modified by experimental design. However, the allocation of this variation to within-site and between-site components depends on how plots and sites are distributed in space. For a fixed spatial pattern of variation, as the distance between plots within a site is increased, the spatial variation within sites increases, and the spatial variation among sites decreases. And if individual sites cover a sufficiently large area, all the spatial variation will reside within sites, and none will reside between sites. This is the sampling design that would maximize statistical power. In fact, if this design were implemented, then pseudoreplication would be eliminated, and it would no longer be necessary to perform a nested ANOVA. However, in practice, it is normally not possible to know that the separation between plots within a site is sufficient to eliminate pseudoreplication, so the nested ANOVA is always advisable. Nonetheless, the power of the nested ANOVA is increased substantially by reducing the spatial structure among sites (Fig. 4), so it is also always advisable to disperse plots over as large an area as possible within a site.

\section{Community Monitoring}

The analyses presented here have been couched in terms of a comparison between independent samples obtained from separate study areas. However, the results apply equally well when the comparison is between samples from the same study area taken at different points in time, as is the case when a community is being monitored. If the samples are independent, that is, if sample sites are located at random during each sampling episode, then all analyses and conclusions apply exactly. However, it is more common to return to the same sampling sites, yielding repeated measures from fixed sites. In this case, the appropriate analysis is a repeated-measures ANOVA, and all conclusions presented above still hold provided that the spatial variance in frequency among sites $\left(\sigma_{s}^{2}\right)$ is replaced with the siteby-time interaction variance. If the pattern of spatial variation in density is highly consistent over time (i.e., there is little or no siteby-time interaction), then the variation among sites will be factored out by the repeated-measures ANOVA, and power will be as high as possible even if plots are tightly packed into sites. At the other extreme, if the pattern of spatial variation in density shows no consistency between sampling periods (i.e., all the spatial variance among sites contributes to the site-by-time interaction variance), then samples from different time periods will be statistically independent, and all conclusions presented in previous paragraphs apply exactly. The degree to which spatial patterns in density will be consistent over time cannot be known in advance, so it is always advisable to disperse plots over as large an area as possible within a site so as to minimize the site-by-time interaction variance.

Even when plots are widely spaced within sites, there can be no assurance that the site-by-time interaction variance has been reduced to 0 , so the repeated-measures ANOVA still must nest plots under sites to avoid pseudoreplication. In such an analysis, the appropriate error variance for testing temporal changes in mean frequency (the main effect of time) is the siteby-time interaction mean square. As a consequence, there is no statistical advantage to obtaining repeated measures from plots within sites. Furthermore, there are well-known disadvantages to fixed plots; relocating small plots can be very difficult and time consuming, and returning to the same plots may cause significant disturbance to the community being monitored. Thus, no extra effort should be expended in relocating plots in successive sampling episodes, and it might even be wise to deliberately relocate plots within the fixed sites.

\section{MANAGEMENT IMPLICATIONS}

Assessment of the effects of specific rangeland management practices requires long-term monitoring of species abundance in conjunction with controlled management protocols. Foliar cover is the most commonly used measure of plant species abundance in large-scale monitoring programs. However, cover may respond strongly to short-term environmental fluctuations, greatly weakening the coupling between cover and density. This decoupling may be a particular problem in grasslands where aboveground biomass is regenerated annually and grazing intensity can be highly variable in space and time. In response to this concern, the Heartland Network Inventory and Monitoring Program within the National Park Service has been conducting a prototype monitoring program at Tallgrass Prairie National Preserve (Cottonwood Falls, KS) in which both frequency and cover are being evaluated as indicators of abundance (http://www1.nature.nps.gov/im/units/htln/index. htm). The present study was undertaken to help guide the design of this prototype program. It is anticipated that plant frequency will be a useful measure of species abundance in other heavily grazed herbaceous communities where long-term monitoring is to be implemented.

For managers who wish to include frequency plots in their monitoring protocol, 3 recommendations for maximizing statistical efficiency emerge from this study: 1) a plot size that returns a mean frequency close to $50 \%$ is nearly optimal over a broad range of values for the magnitude of spatial structure; 2) frequency plots within the same site (often located along transects) should be dispersed over as large an area as possible; and 3) study sites, but not individual frequency plots, should be permanently marked and resampled over time. 


\section{ACKNOWLEDGMENTS}

We thank 3 anonymous reviewers for many valuable suggestions that have been incorporated into this manuscript.

\section{LITERATURE CITED}

Bonham, C. D. 1989. Measurements for terrestrial vegetation. New York, NY: John Wiley and Sons. $338 \mathrm{p}$.

Causton, D. R. 1988. An introduction to vegetation analysis. London, United Kingdom: Unwin Hyman. $342 \mathrm{p}$.

Critchley, D. N. R., and S. M. C. Poulton. 1998. A method to optimize precision and scale in grassland monitoring. Journal of Vegetation Science 9:837-846.

CuRTIS, J. T., AND R. P. MclNTOSH. 1950. The interrelations of certain analytic and synthetic phytosociological characters. Ecology 31:434-455.

DeBacker, M. D., A. N. Sasseen, C. Becker, G. A. Rowell, L. P. Thomas, J. R. Boetsch, AND G. D. WiLLSON. 2004. Vegetation community monitoring protocol for the Heartland I\&M Network and Prairie Cluster prototype monitoring program. Republic, MO: National Park Service. 213 p.

Fonteyn, P. J., and B. E. Mahall. 1981. An experimental analysis of structure in a desert plant community. Journal of Ecology 69:883-896.

GreIG-Smith, P. 1983. Quantitative plant ecology. 3rd ed. Berkeley: University of California Press. 359 p.

Hartnett, D. C., and P. A. Fay. 1998. Plant populations: patterns and process. In: A. K. Knapp, J. M. Briggs, D. C. Hartnett, and S. L. Collins [EDs.]. Grassland dynamics: Long-term ecological research in tallgrass prairie. New York, NY: Oxford University Press. p. 81-100.

Hyder, D. N., R. E. Bement, E. E. Remmenga, and C. Terwilliger, JR. 1965. Frequency sampling of blue grama range. Journal of Range Management 18:90-94.
Hyder, D. N., R. E. Bement, E. E. Remmenga, and C. Terwilliger, JR. 1966. Vegetationsoils and vegetation-grazing relations from frequency data. Journal of Range Management 19:11-17.

Hyder, D. N., C. E. Conrad, P. T. Tueller, L. D. Calvin, C. E. Poulton, and F. A. Sneva. 1963. Frequency sampling in sagebrush-bunchgrass vegetation. Ecology 44:740-746.

KerShaw, K. A., and J. H. H. LoOney. 1985. Quantitative and dynamic plant ecology. 3rd ed. London, United Kingdom: Edward Arnold. 282 p.

LesicA, P., AND D. HANNA. 2002. Monitoring composition of foothills grassland using frequency of indicator species. Natural Areas Journal 22:148-153.

Morrison, D. A., A. F. Le Brocque, and P. J. Clarke. 1995. An assessment of some improved techniques for estimating the abundance (frequency) of sedentary organisms. Vegetatio 120:131-145.

Mosley, J. C., S. C. Bunting, and M. Hironaka. 1986. Determining range condition from frequency data in mountain meadows of central Idaho. Journal of Range Management 39:561-565.

Mueller-Dombois, D., and H. Ellenburg. 1974. Aims and methods of vegetation ecology. New York, NY: John Wiley and Sons. 547 p.

Outhred, R. K. 1984. Semi-quantitative sampling in vegetation survey. In: K. Myers, C. R. Margules, and I. Musto [EDS.]. Survey methods for nature conservation. Canberra, Australia: CSIR0. p. 87-100.

Peet, R. K., J. Wentworth, and P. White. 1998. A flexible multipurpose method for recording vegetation composition and structure. Castanea 63:262-274.

Smith, S. D., S. C. Bunting, and M. Hironaka. 1986. Sensitivity of frequency plots for detecting vegetation change. Northwest Science 60:279-286.

Smith, S. D., S. C. Bunting, and M. Hironaka. 1987. Evaluation of the improvement in sensitivity of nested frequency plots to vegetation change by summation. Great Basin Naturalist 47:299-307.

Whysong, G. L., And W. W. Brady. 1987. Frequency sampling and type II errors. Journal of Range Management 40:472-474. 